\title{
Comparison of Dynamic Contrast-Enhanced 3T MR and 64-Row Multidetector CT Angiography for the Localization of Spinal Dural Arteriovenous Fistulas
}

\author{
S. Oda, D. Utsunomiya, T. Hirai, Y. Kai, Y. Ohmori, Y. Shigematsu, Y. Iryo, H. Uetani, M. Azuma, and Y. Yamashita
}

\begin{abstract}
BACKGROUND AND PURPOSE: For the localization of spinal dural arteriovenous fistulas, it is not determined whether dynamic contrast-enhanced MRA is more reliable than multidetector CTA. The aim of this study was to compare the agreement between intra-arterial DSA, dynamic contrast-enhanced MRA at 3T, and 64-row multidetector CTA for the localization of spinal dural arteriovenous fistulas.

MATERIALS AND METHODS: We enrolled 12 consecutive patients (11 men, 1 woman; age range, 46-83 years; mean, 65 years) who underwent preoperative dynamic contrast-enhanced MRA at 3T and 64-row multidetector CTA. The spinal dural arteriovenous fistula location was confirmed by intra-arterial DSA as the reference standard. Two reviewers independently evaluated the level of the artery feeding the spinal dural arteriovenous fistula on the basis of continuity between the feeder and abnormal spinal vessels on 3T dynamic contrast-enhanced MRA and 64-row multidetector CTA images. Interobserver and intermodality agreement was determined by calculation of the $\kappa$ coefficient.
\end{abstract}

RESULTS: On DSA, the vessel feeding the spinal dural arteriovenous fistula was the intercostal artery ( 7 cases), the lumbar artery ( 3 cases), and the internal iliac artery or the ascending pharyngeal artery (1 case each). For the fistula level, interobserver agreement was excellent for $3 \mathrm{~T}$ dynamic contrast-enhanced MRA $(\kappa=0.97 ; 95 \% \mathrm{Cl}, 0.92-1.00)$ and very good for 64-row multidetector $\mathrm{CTA}(\kappa=0.84 ; 95 \% \mathrm{Cl}$, $0.72-0.96)$. Intermodality agreement with DSA was good for 3 T dynamic contrast-enhanced MRA $(\kappa=0.78 ; 95 \% \mathrm{Cl}, 0.49-1.00)$ and moderate for 64-row multidetector CTA $(\kappa=0.41 ; 95 \% \mathrm{Cl}, 0.020-0.84)$.

CONCLUSIONS: For the localization of spinal dural arteriovenous fistulas, 3T dynamic contrast-enhanced MRA may be more reliable than 64-row multidetector CTA.

ABBREVIATIONS: DCE = dynamic contrast-enhanced; SDAVF = spinal dural arteriovenous fistulas; $\mathrm{VR}=$ volume-rendering; $64-\mathrm{CTA}=64-$ row multidetector CTA

$\mathbf{S}$ pinal dural arteriovenous fistulas (SDAVF) are the most commonly encountered spinal vascular shunt lesions and a treatable cause of myelopathy. ${ }^{1}$ The arteriovenous shunt is located inside the dura mater close to the spinal nerve root, where the arterial blood from a radiculomeningeal artery enters a radicular vein. Shunt interruption by either neurosurgery or superselective embolization is the basic treatment strategy, ${ }^{1-3}$ and pretreatment localization of SDAVF is important for their adequate treatment. Catheter spinal DSA is the standard technique that accurately

Received March 20, 2013; accepted April 18

From the Departments of Diagnostic Radiology (S.O., D.U., T.H., Y.S., Y.I., H.U., M.A., Y.Y.) and Neurosurgery (Y.K., Y.O.), Graduate School of Medical Sciences, Kumamoto University, Kumamoto, Japan.

Please address correspondence to Toshinori Hirai, MD, Department of Diagnostic Radiology, Graduate School of Medical Sciences, Kumamoto University, 1-1-1 Honjo, Kumamoto 860-8556, Japan; e-mail: t-hirai@kumamoto-u.ac.jp

http://dx.doi.org/10.3174/ajnr.A3660 detects the location of SDAVF and their feeders. However, an exhaustive search for the lesion by selective catheterization is time-consuming and increases the amount of radiation exposure and contrast material and is accompanied by risks for neurologic complications. $^{4,5}$

Noninvasive imaging modalities such as multidetector CTA and dynamic contrast-enhanced (DCE)-MRA reliably detect SDAVF and may predict the level of their location. ${ }^{5-11}$ However, the noninvasive technique more reliable for the localization of SDAVF remains to be identified, and interobserver and intermodality agreement for the location of SDAVF on CTA and DCEMRA studies has not been fully investigated.

In multidetector CTA, scanner performance can be improved by adding detector rows, ${ }^{12}$ and, in DCE-MRA, higher magnetic fields improve the image quality while reducing the acquisition time. ${ }^{13}$ In the present study, we compared the agreement between DSA, DCE-MRA at 3T, and 64-row multidetector CTA (64-CTA) for the localization of SDAVF. 
Table 1: Summary of patients and SDAVF

\begin{tabular}{ccccccc}
\hline Case No. & Age, $\boldsymbol{y}$ & Sex & $\begin{array}{c}\text { Clinical } \\
\text { Manifestation }\end{array}$ & $\begin{array}{c}\text { Extent of Cord } \\
\text { Signal Abnormality }\end{array}$ & Feeder & Fistula Level \\
\hline 1 & 46 & M & Paraparesis & C2-C7 & L, APhA & FM \\
2 & 58 & M & Paraplegia & T3-CM & L, IA & T5 \\
3 & 75 & M & Paraparesis & T7-CM & R, IA & T6 \\
4 & 60 & M & Paraplegia & T8-CM & R, IA & T7 \\
5 & 58 & M & Paraparesis & T5-CM & R, IA & T7 \\
6 & 78 & M & Paraplegia & T7-T11 & L, IA & T7 \\
7 & 62 & M & Paraplegia & T5-CM & R, IA & T8 \\
8 & 75 & F & Quadriparesis & C5-C6 & R, IA & T9 \\
9 & 76 & M & Paraplegia & T6-CM & R, LA & L2 \\
10 & 83 & M & Paraplegia & T3-CM & R, LA & L2 \\
11 & 58 & M & Paraplegia & T5-CM & L, LA & L3 \\
12 & 52 & M & Paraplegia & T3-CM & L, IIA & S1 \\
\hline
\end{tabular}

Note:-L indicates left; R, right; T, thoracic spine; L, lumbar spine; S, sacral spine; CM, conus medularis; APhA, ascending pharyngeal artery; IA, intercostal artery; LA, lumbar artery; IIA, internal iliac artery; FM, foramen magnum.
To obtain 3D images of the vessels injected from the feeder and of the bony structures, we acquired 3D rotational angiographs by use of the same angiography system. The parameters were 4.1-second rotation; rotation angle, $240^{\circ}$ with $2^{\circ}$ increments, resulting in 120 projections; rotation speed, $55^{\circ} / \mathrm{s}$, acquisition matrix, $1024 \times 1024$; frame rate, 30 frames/s. The volume and the injection rate of the nonionic iodinated contrast agent were $15 \mathrm{~mL}$ and $1.5 \mathrm{~mL} / \mathrm{s}$ respectively. We then reconstructed and analyzed the filling run-volume by use of a dedicated commercially available workstation (Philips Healthcare). The 3D images were recon-

\section{MATERIALS AND METHODS}

\section{Study Population}

Our study was approved by our institutional review board. Prior informed consent for imaging examinations was obtained from all patients or their relatives. Between October 2008 and December 2012, 23 patients were referred for spinal 64-CTA and DCEMRA at 3T and for the evaluation of possible SDAVF suspected on the basis of combined spinal MR imaging and clinical findings. Our inclusion criteria were a diagnosis of SDAVF on the basis of spinal DSA scans and verified at surgery after spinal 3T DCEMRA and 64-CTA examinations. Exclusion criteria were renal dysfunction (estimated glomerular filtration rate $<30 \mathrm{~mL} / \mathrm{min}$ per $1.73 \mathrm{~m}^{2}$ ) and allergy to iodinated and gadolinium-based contrast materials. On the basis of these criteria, we enrolled 12 consecutive patients (11 men and 1 woman ranging in age from 46-83 years; mean age, 65 years). All patients presented with congestive myelopathy and a diffuse, continuous hyperintense cord lesion in various cord regions (Table 1), and all underwent spinal CTA, MRA, and intra-arterial DSA. The interval between CTA, MRA, and DSA studies ranged from 3-20 days (mean, 10 days).

\section{DSA Technique}

Diagnostic intra-arterial DSA through a femoral arterial approach was performed in a biplane angiography suite (Allura Xper FD; Philips Healthcare, Best, the Netherlands) by a trained neuroradiologist and/or a neurosurgeon. The angiographic technique included the selective manual injection of $3-5 \mathrm{~mL}$ of a $300-\mathrm{mg} / \mathrm{mL}$ iodinated nonionic contrast agent into the intended arteries and anteroposterior imaging at a rate of 3 frames/s. Images were obtained with a $2048 \times 2048$ matrix; the FOV was $42 \mathrm{~cm}$.

When DCE-MRA and CTA findings suggested the location of the fistula, we first delivered a selective manual injection at the anticipated level. If the fistula was identified, the contralateral segmental artery and the segmental arteries ranging from 2 levels above to 2 levels below the fistula were studied to ensure complete evaluation of the fistula and the adjacent vasculature. If the fistula site was not identified, additional injections were delivered into segmental arteries from the supreme intercostal artery to the median sacral arteries, the bilateral internal iliac arteries, and the bilateral vertebral, subclavian, costocervical, thyrocervical, and external carotid arteries. structed in a $512 \times 512 \times 512$ matrix with an isotropic voxel size.

\section{CT Data Acquisition}

All CT studies were performed on a 64-detector CT system (Brilliance-64, Philips Healthcare) with a 0.5 -second gantry rotation speed, an $\mathrm{x}$-ray tube voltage of $120 \mathrm{kV}$, and an $\mathrm{x}$-ray tube current of $412 \mathrm{~mA}$. The collimation was $64 \times 0.625 \mathrm{~mm}$, the beam pitch was 0.515 , and the table speed was $20.6 \mathrm{~mm}$ per rotation. A double-head power injector (Dual Shot-Type GX; Nemoto Kyorindo, Tokyo, Japan) was used to administer a bolus of $350 \mathrm{mgI} / \mathrm{mL}$ contrast medium (135 mL of iomeprol, Iomeron; Bracco, Milan, Italy) at $5.0 \mathrm{~mL} / \mathrm{s}$ through a 20 -gauge IV catheter in an antecubital vein; the contrast agent was followed by a $40-\mathrm{mL}$ bolus of saline solution at the same rate.

Synchronization between the flow of contrast material and CT acquisition was achieved by use of a computer-assisted bolus tracking system. The CT attenuation value was monitored by a radiology technologist. The anatomic level for monitoring was set in the descending aorta at the T10 level on the scout CT image. The trigger threshold was set at $250 \mathrm{HU}$ for the aortic ROI. CT data acquisition was started 10 seconds after triggering. Data were acquired during a single breath-hold in the head-to-foot direction. The CT scan was from the level of the foramen magnum to the groin.

The helical data were reconstructed in the axial plane with a $0.5-\mathrm{mm}$ section thickness at $0.3-\mathrm{mm}$ intervals before storage and transfer to a workstation (M900QUADRA; Amin, Tokyo, Japan). The multiplanar reformation images, including oblique coronal images with craniocaudal angulations and curved planar reformation images, were reconstructed at a voxel size of $0.4 \times 0.4 \times 0.7 \mathrm{~mm}$ to confer the greatest possible likelihood that the spinal vessels were included in the scan. Volume-rendering (VR) CTA images were also reconstructed for image interpretation.

\section{MR Data Acquisition}

All MR studies were performed on a 3T MR imaging system (Magnetom Trio, Siemens, Erlangen, Germany) equipped with a phased-array spine coil. The patients were imaged in the supine 
position with a 20-gauge intravenous catheter inserted into the antecubital vein. Conventional MR imaging was with sagittal and axial T1- and T2-weighted sequences.

A test bolus was delivered to determine the arrival time of the contrast agent in the arteries feeding the spine. The intravenous injection of $0.2 \mathrm{~mL}$ gadopentetate dimeglumine (Magnevist; Bayer HealthCare Pharmaceuticals, Wayne, New Jersey) per kilogram of body weight, delivered at a flow rate of $3 \mathrm{~mL} / \mathrm{s}$, was followed by a $30-\mathrm{mL}$ saline flush administered with an automated power injector. On the basis of test bolus results, we injected the contrast agent and started dynamic 3-phase DCE-MRA with a 3D FLASH sequence in the coronal plane. To facilitate subtraction DCE-MRA, 1 precontrast phase, composed of the exact same pulse sequence parameters as the DCE-MRA sequence, was acquired. The imaging data were acquired during breath-holding. The FOV of contrast-enhanced MRA was positioned to cover the entire T2 hyperintense cord and dilated vessels. If previous contrast-enhanced MR images were available, they were also used for determining the positioning of the FOV. The acquisition parameters for the contrast-enhanced MRA sequence were TR/TE, 3.1 $\mathrm{ms} / 1.1 \mathrm{~ms}$; flip angle, $15^{\circ}$; image matrix, $280 \times 390$; FOV, 400 $\mathrm{mm}$; slab thickness, $78 \mathrm{~mm}$; generalized autocalibrating partially parallel acquisition, 2 . The reconstructed voxel size and temporal resolution were $1.4 \times 1.2 \times 1.5 \mathrm{~mm}$ and 17 seconds, respectively. Subtracted maximum-intensity projection, partial MIP, and VR DCE-MRA images in arterial and venous phases and their MPR images were reconstructed for image interpretation.

\section{Image Evaluation}

Two independent readers (Y.K. and Y.O., with 23 and 14 years of experience in neuroangiography, respectively) qualitatively evaluated the entire series of DSA images on a PACS workstation. Disagreements were resolved by consensus. Two other readers (T.H. and Y.I., with 21 and 8 years of experience in diagnostic neuro-MR imaging, respectively), blinded to the clinical, CTA, and DSA results, independently evaluated the DCE-MRA data on a PACS workstation. In each case, the subtracted source, MPR, MIP, partial MIP, and VR DCE-MRA images and conventional MR imaging data were displayed with all regions visible. Two other readers (D.U. and S.O., with 16 and 8 years of experience in CTA, respectively), blinded to the clinical, DCE-MRA, and DSA results, independently evaluated the CTA data on a PACS workstation. In each case, the source, MPR, and VR CTA images were displayed with all regions visible. Our software allowed the enlargement of regions of special interest in any given spatial orientation.

Each reviewer for DSA, DCE-MRA, and CTA recorded the shunt level of the SDAVF on the basis of continuity between the feeding artery and abnormal spinal vessels. When the 2 reviewers disagreed, final determinations were based on consensus readings.

After the blinded study, the observers consensually reviewed the reasons of incorrect interpretation for the fistula location with the DSA findings. The observers also determined whether CTA provided additional information to DCE-MRA with regard to the fistula location of SDAVF.
Table 2: Summary of localization of SDAVF on DCE-MRA and DSA

\begin{tabular}{|c|c|c|c|c|c|c|}
\hline \multirow{2}{*}{$\begin{array}{l}\text { Case } \\
\text { No. }\end{array}$} & \multicolumn{2}{|c|}{ DCE-MRA } & \multirow{2}{*}{$\begin{array}{l}\text { Interobserver } \\
\text { Agreement }^{\mathrm{a}}\end{array}$} & \multirow{2}{*}{$\begin{array}{l}\text { DCE- } \\
\text { MRA }^{\mathrm{b}}\end{array}$} & \multirow[b]{2}{*}{ DSA } & \multirow{2}{*}{$\begin{array}{l}\text { Intermodality } \\
\text { Agreement }^{c}\end{array}$} \\
\hline & Reader 1 & Reader 2 & & & & \\
\hline 1 & $\mathrm{FM}$ & $\mathrm{FM}$ & & $\mathrm{FM}$ & $\mathrm{FM}$ & \\
\hline 2 & T5 & $\mathrm{T} 5$ & & T5 & T5 & \\
\hline 3 & T6 & T6 & & T6 & T6 & \\
\hline 4 & $\mathrm{~T} 7$ & $\mathrm{~T} 7$ & & $\mathrm{~T} 7$ & $\mathrm{~T} 7$ & \\
\hline 5 & $\mathrm{~T} 7$ & $\mathrm{~T} 7$ & & $\mathrm{~T} 7$ & $\mathrm{~T} 7$ & \\
\hline 6 & $\mathrm{~T} 10$ & $\mathrm{~T} 70$ & 11 (92\%) & $\mathrm{T} 70$ & $\mathrm{~T} 7$ & 10 (83\%) \\
\hline 7 & T8 & T8 & $\kappa=0.97$ & $\mathrm{~T} 8$ & T8 & $\kappa=0.78$ \\
\hline 8 & L2 & L3 & [0.92-1.00] & L2 & T9 & [0.49-1.00] \\
\hline 9 & L2 & L2 & & L2 & L2 & \\
\hline 10 & L2 & L2 & & L2 & L2 & \\
\hline 11 & L3 & L3 & & L3 & L3 & \\
\hline 12 & S1 & $\mathrm{S} 1$ & & $\mathrm{S1}$ & $\mathrm{S1}$ & \\
\hline
\end{tabular}

Note:-Data are number of cases. Data in parentheses are the percentage of times that results were concordant; data in brackets are $95 \% \mathrm{Cls}$.

FM indicates foramen magnum; T, thoracic spine; L, lumbar spine; S, sacral spine.

${ }^{a}$ Agreement of DCE-MRA between reader 1 and reader 2 .

${ }^{\mathrm{b}}$ Consensus reading of DCE-MRA of reader 1 and reader 2 .

${ }^{c}$ Agreement between the consensus reading of DCE-MRA of reader 1 and reader 2 and DSA.

\section{Statistical Analysis}

The level of interobserver agreement (between readers 1 and 2 for DSA, DCE-MRA, and CTA) and of intermodality agreement (between consensus readings of DCE-MRA/CTA and DSA images) with respect to the location of the SDAVF was determined by calculating the $\kappa$ coefficient $(\kappa<0.20$, poor; $\kappa=0.21-0.40$, fair; $\kappa=0.41-0.60$, moderate; $\kappa=0.61-0.80$, good; $\kappa=0.81-0.90$, very good; and $\kappa>0.90$, excellent agreement) with a $95 \%$ CI. The laterality of the fistula site was not put into the statistical analyses. We also recorded the exact number and percentage of times when the results from both readers and both modalities were in exact agreement. MedCalc for Windows (MedCalc Software, Mariakerke, Belgium) was used for all analyses.

\section{RESULTS}

DCE-MRA, 64-CTA, and intra-arterial DSA were performed successfully in all 12 patients. At qualitative evaluation of DSA, interobserver agreement for the fistula location was excellent $(\kappa=1.0$; $95 \%$ CI, 1.0-1.0). In 7 cases, the location of the SDAVF was the thoracic spine (T5, T6, T8, T9, $n=1$ each; T7, $n=3$ ); in 3 it was the lumbar spine (L2, $n=2 ; \mathrm{L} 3, n=1)$. In the other 2 cases, the SDAVF was located at the sacral (S1) or the cervical spine (foramen magnum). The locations of the feeders and fistulas identified on DSA images are shown in Table 1.

Table 2 is a summary of DCE-MRA and DSA findings. In 11 of 12 cases (92\%), both readers agreed on the fistula location on DCE-MRA images, and interobserver agreement was excellent $(\kappa=0.97 ; 95 \%$ CI, 0.92-1.00; Figs 1, 2, and 3). In 10 of 12 studies $(83 \%)$, the DCE-MRA (consensus reading) and DSA findings of both readers coincided with respect to the fistula location. Intermodality agreement (DCE-MRA versus DSA findings) was good $(\kappa=0.78 ; 95 \%$ CI, 0.49-1.00).

As shown in Table 3, both readers reviewing CTA images agreed on the fistula location in 7 of 12 studies (58\%); interobserver agreement was very good $(\kappa=0.84 ; 95 \%$ CI, 0.72-0.96; Figs $1-3)$. In 7 of 12 studies (58\%), the CTA (consensus reading) and DSA findings of both readers coincided with respect to the fistula location. Intermodality agreement (CTA versus DSA findings) was moderate ( $\kappa=0.41 ; 95 \%$ CI, $0.020-0.84)$. 

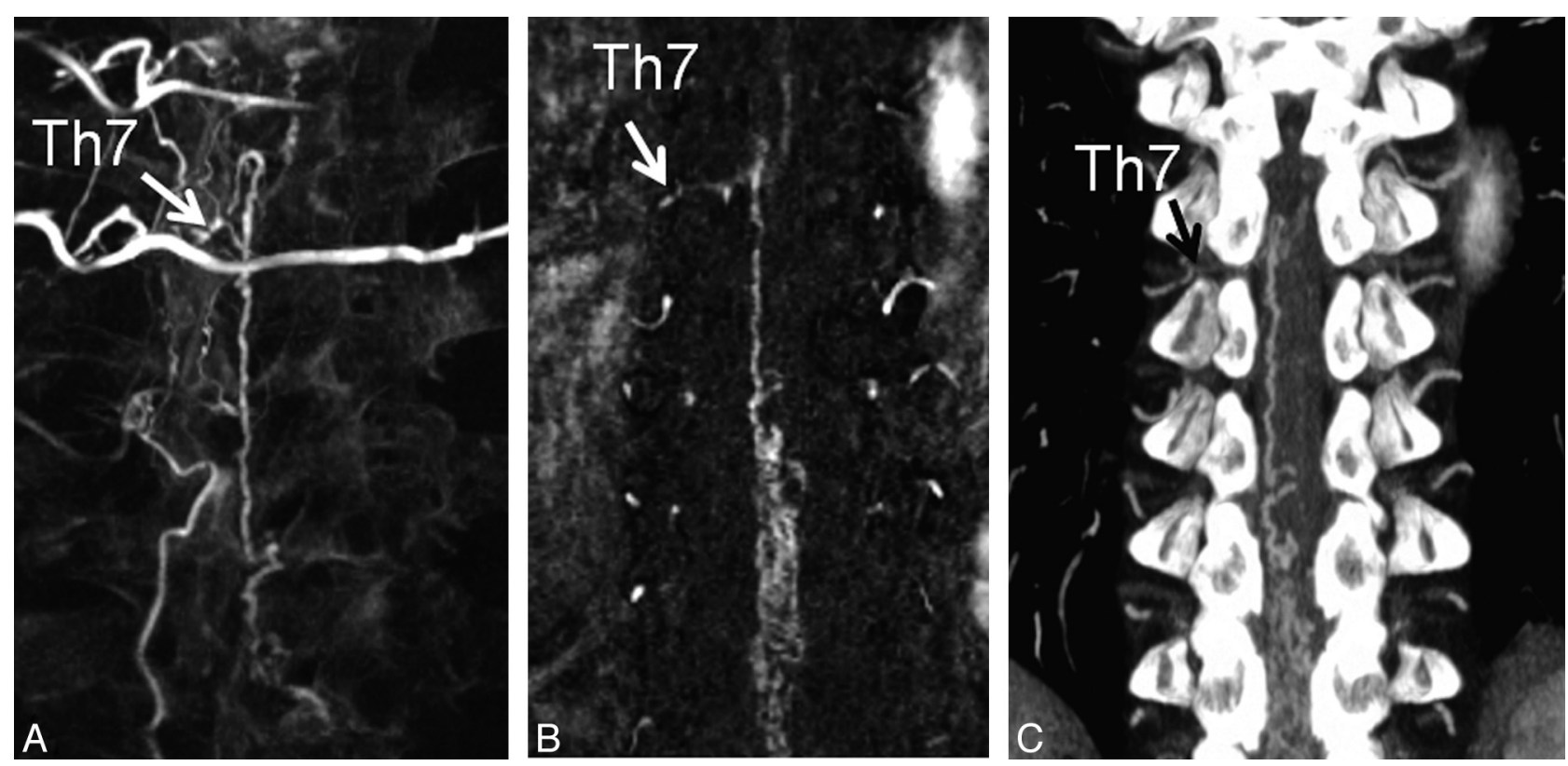

FIG 1. A 58-year-old man (case 5) with SDAVF at the T7 level. A, Reconstructed coronal MPR 3D angiogram demonstrates the fistula site at the level of T7 on the right side (arrow) and the surrounding vertebrae. B, Subtracted coronal partial-MIP DCE-MRA image shows the fistula site at the level of $\mathrm{T} 7$ on the right side (arrow). Two readers judged the fistula location as T7. C, On the coronal partial-MIP CTA image, the feeding artery of the SDAVF is depicted to derive from the 7th thoracic level (T7) on the right side (arrow). Two readers judged the fistula location as T7.
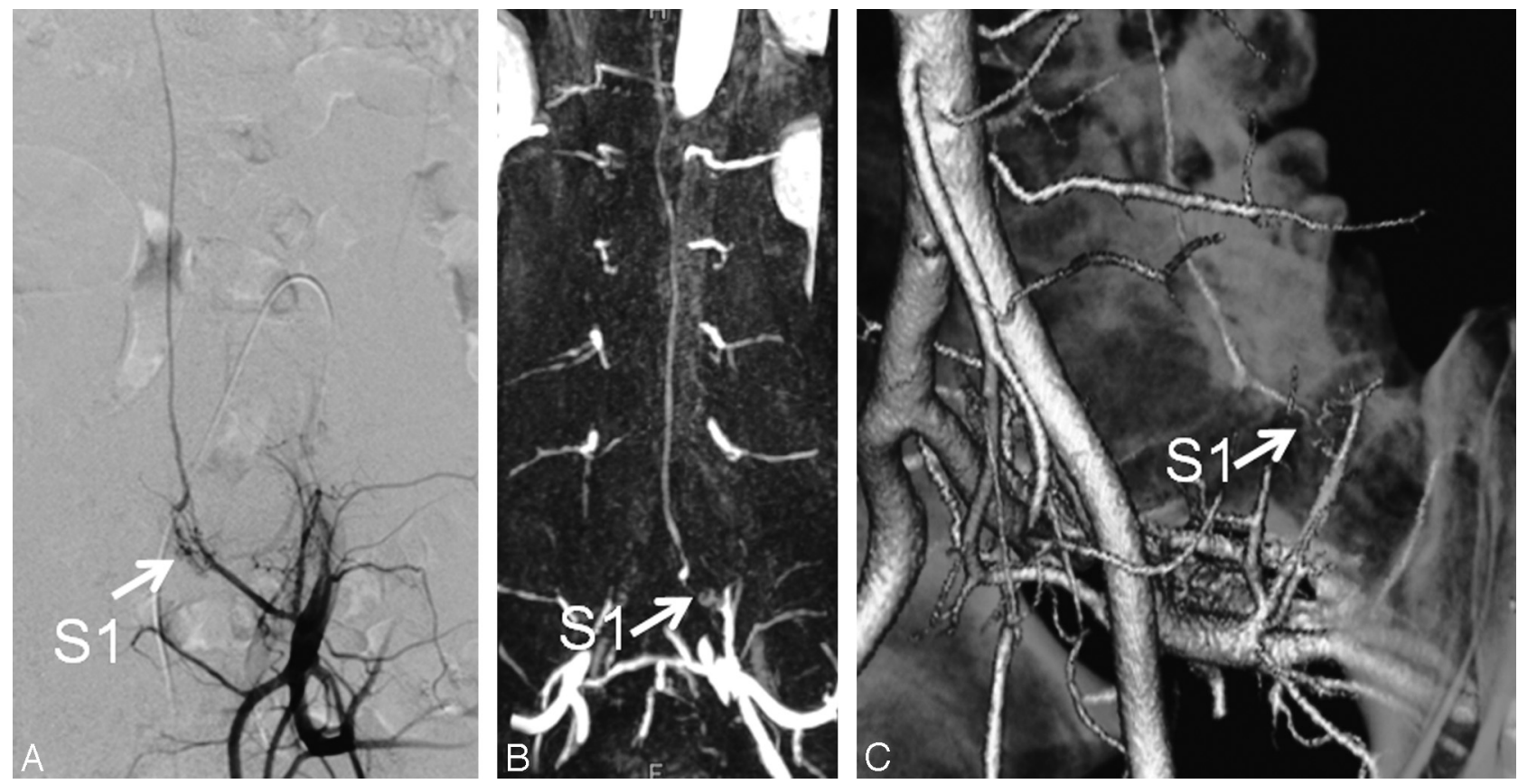

FIG 2. A 52-year-old man (case 12) with SDAVF at the S1 level. A, Catheter angiogram from the left internal iliac artery depicts the fistula site at the level of S1 (arrow). B, Subtracted coronal partial-MIP DCE-MRA image shows the fistula site at the S1 level (arrow). Two readers judged the fistula location as S1. C, On the oblique VR CTA image, 2 readers judged the fistula level to be at S1 (arrows). In the retrospective consensus review, additional use of CTA images increased the observers' confidence level of the diagnosis.

In the retrospective consensus reviews, the incorrect interpretation on DCE-MRA and CTA was found in 2 of 12 (17\%) and 5 of $12(42 \%)$, respectively. The reasons for the incorrect interpretation on DCE-MRA in the 2 cases were considered to be small caliber of the feeder and the radicular vein at the spinal nerve root. In the 5 cases on CTA, the incorrect interpretation was caused by the overlap of bony structures and the vessels at the spinal nerve root in 3 cases, the scanning of inappropriate arterial phase in 1 case (Fig 3), and the presence of slight venous contamination in 1 case. Although there were no cases in which the observer's judgment for diagnosing the fistula location of SDAVF was changed by CTA information, their confidence level in the diagnosis was further increased by use of combined DCE-MRA and CTA images in 3 cases. 

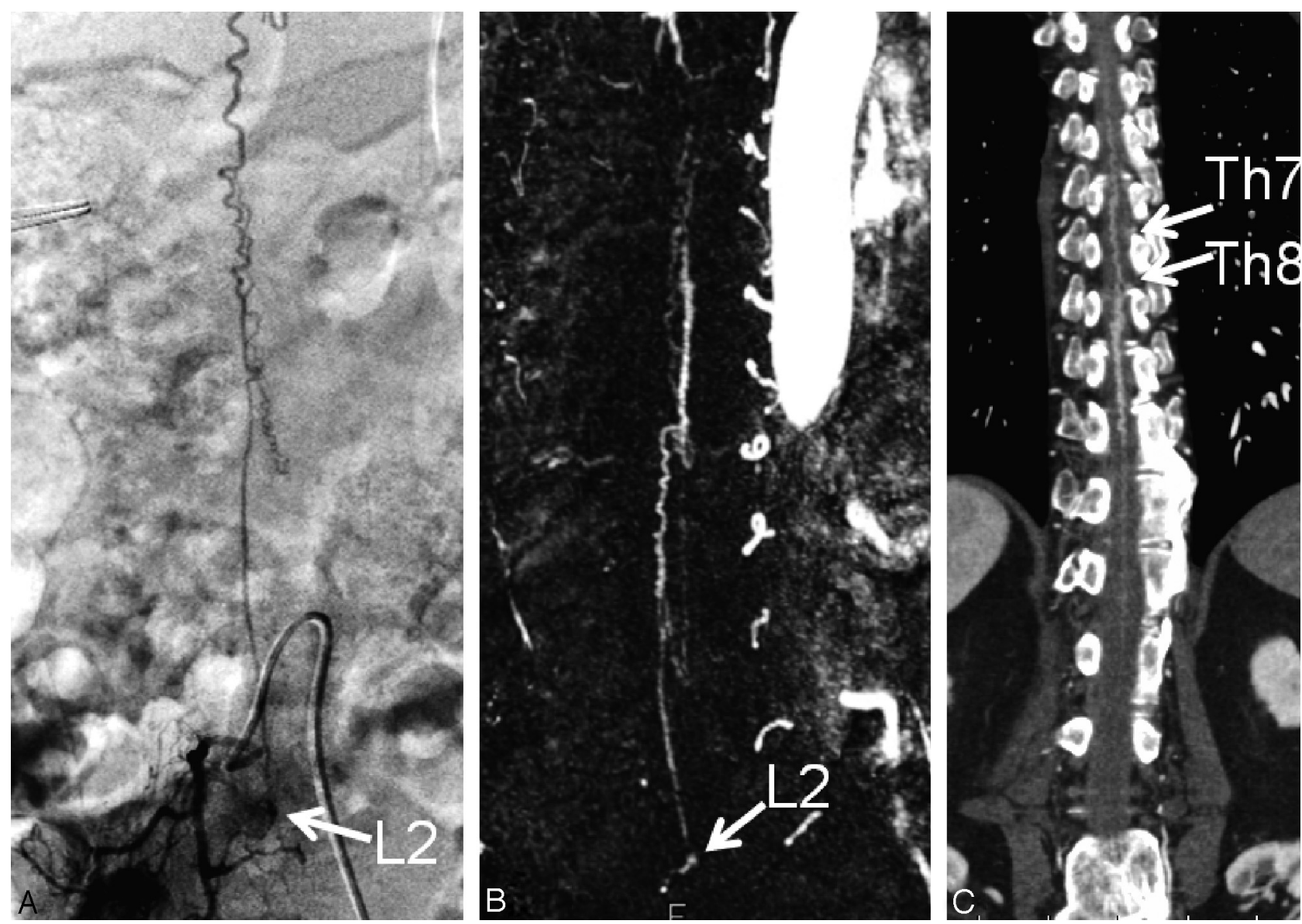

FIG 3. A 76-year-old man (case 9) with SDAVF at the L2 level. A, Catheter angiogram from the right lumbar artery demonstrates the fistula site at the level of L2 on the right side (arrow). B, Subtracted coronal partial-MIP DCE-MRA image shows the fistula site at the L2 level (arrow). Two readers judged the fistula location as L2. C, On the coronal partial-MIP CTA image, 2 readers judged the fistula level to be at T7 and T8, respectively (arrows). In the retrospective consensus review, the reason for their incorrect interpretation was considered to be scanning of inappropriate arterial phase of CTA.

Table 3: Summary of localization of SDAVF at CTA and DSA

\begin{tabular}{|c|c|c|c|c|c|c|}
\hline \multirow{2}{*}{$\begin{array}{l}\text { Case } \\
\text { No. }\end{array}$} & \multicolumn{2}{|c|}{ CTA } & \multirow{2}{*}{$\begin{array}{l}\text { Interobserver } \\
\text { Agreement }^{\mathrm{a}}\end{array}$} & \multirow[b]{2}{*}{$\mathrm{CTA}^{\mathrm{b}}$} & \multirow[b]{2}{*}{ DSA } & \multirow{2}{*}{$\begin{array}{l}\text { Intermodality } \\
\text { Agreement }^{c}\end{array}$} \\
\hline & Reader 1 & Reader 2 & & & & \\
\hline 1 & FM & FM & & $F M$ & FM & \\
\hline 2 & T5 & T5 & & T5 & T5 & \\
\hline 3 & T6 & T6 & & T6 & T6 & \\
\hline 4 & T8 & T9 & & T9 & $\mathrm{T7}$ & \\
\hline 5 & $\mathrm{T7}$ & $T 7$ & & 77 & $T 7$ & \\
\hline 6 & $\mathrm{~T} 10$ & $\mathrm{~T} 12$ & $7(58 \%)$ & $\mathrm{T} 12$ & $\mathrm{T7}$ & $7(58 \%)$ \\
\hline 7 & T8 & T8 & $\kappa=0.84$ & T8 & T8 & $\kappa=0.41$ \\
\hline 8 & L2 & L3 & {$[0.72-0.96]$} & L2 & T9 & {$[0.02-0.84]$} \\
\hline 9 & T8 & $\mathrm{T7}$ & & T8 & L2 & \\
\hline 10 & T9 & T8 & & T9 & L2 & \\
\hline 11 & L3 & L3 & & L3 & L3 & \\
\hline 12 & $\mathrm{~S} 1$ & $\mathrm{~S} 1$ & & $\mathrm{~S} 1$ & $\mathrm{~S} 1$ & \\
\hline
\end{tabular}

Note:-Data are number of cases. Data in parentheses are the percentage of times that results were concordant; data in brackets are $95 \% \mathrm{Cls}$.

FM indicates foramen magnum; T, thoracic spine; L, lumbar spine; S, sacral spine.

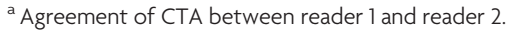

${ }^{b}$ Consensus reading at CTA of reader 1 and reader 2 .

${ }^{c}$ Agreement between the consensus reading at CTA of reader 1 and reader 2 and DSA.

\section{DISCUSSION}

Our study showed that for the characterization of SDAVF, DCEMRA at 3T was more reliable than 64-CTA. We attribute the good interobserver and intermodality agreement for DCE-MRA to 3 factors. First, we performed dynamic 3-phase contrast-enhanced MRA with a parallel imaging technique. The DCE-MRA images were acquired with the patients holding their breath, and this allowed data acquisition with a temporal resolution of $17 \mathrm{~s} / \mathrm{vol}-$ ume, at which it was possible to differentiate the arterial and venous phases. Second, because we used a high-field 3T MR imaging unit, our DCE-MRA studies benefited from 2 key conditions. Namely, the theoretic signal-to-noise ratio at $3 \mathrm{~T}$ is twice that at $1.5 \mathrm{~T}$, thus allowing for increased spatial resolution. The longer $\mathrm{T} 1$ values of tissues at $3 \mathrm{~T}$ yield better background suppression and contrast-to-noise ratios. ${ }^{13-15}$ Third, the DCE-MRA images that we used provided a uniform background from the subtraction technique, and this rendered the detection of subtle vascular lesions easier.

The spatial resolution was higher on 64-CTA than on 3T DCEMRA studies; however, intermodality agreement for the identification of the location of the SDAVF was insufficient. In our study, adequate arterial phase on CTA was not obtained in 2 (17\%) of 12 cases. In our protocol, CT acquisition was performed by use of a computer-assisted bolus tracking system, and its monitoring was set in the descending aorta at the T10 level. However, the level of feeders of SDAVF was variable: cervical to sacral vertebrae. Therefore, adequate arterial phase on CTA may not always have been obtained. The identification of feeding arteries and radicular veins adjacent to the bones may have been hampered because of the 
similar attenuation of these structures. In our retrospective reviews, the incorrect interpretation from this effect was found in 3 (25\%) of 12 cases. Background suppression of bones can be achieved on CTA scans by first acquiring a precontrast scan followed by subtraction from the contrast-enhanced scan. However, this would further increase radiation exposure and the noise level.

Zampakis et $\mathrm{al}^{7}$ reported that the sensitivity of MRA and CTA for determining the level of SDAVF was $40 \%$ and $90 \%$, respectively. Their results differ from ours. They used 2 different contrast-enhanced MRA techniques at 1.5T, that is, a first-pass elliptical centric contrast-enhanced MRA technique in 7 patients and an elliptical centric time-resolved imaging in constant kinetics technique in 2 patients. They obtained single 3D volume data at an acquisition time of 52 seconds with the former and $203 \mathrm{D}$ volume datasets at 1.7 seconds of temporal resolution with the latter technique, and they did not use subtraction. Under these imaging conditions for contrast-enhanced MRA, they may not have used the appropriate arterial phase for spinal MRA scans, and this may have rendered difficult the interpretation of their unsubtracted MRA images.

Compared with DCE-MRA, multidetector CTA generally provides for a shorter scan time, larger scan coverage, and higher spatial resolution and facilitates the observation of enhanced vessels with the surrounding bony structures. In our study, the observer's confidence level of the diagnosis was furthermore increased by additional use of CTA in 3 (25\%) of 12 cases. This is thought to be a benefit for planning of diagnostic and interventional spinal angiography. For surgical planning, the visualization on CTA images of both vessels and bones may be advantageous. On the other hand, a distinct disadvantage of CTA is the patient exposure to ionizing radiation. A CT scan of the entire spinal cord delivers an effective dose of approximately $20 \mathrm{mSv} .{ }^{16}$ To achieve a radiation dose reduction, CT scanners with more detectors (eg, 256- or 320-detector CT scanners) and iterative reconstruction algorithms might be useful. ${ }^{17}$ Further experimental and clinical studies are required to clarify this issue.

Our study has some limitations. First, we did not compare different 3D reconstructing techniques (eg, VR, MIP) for the display of SDAVF on DCE-MRA and CTA images. Although the most suitable display method for evaluating SDAVF on DCEMRA and CTA scans remains to be identified, we used source, MPR, MIP, and partial MIP images with or without VR images. These are widely used in the clinical setting to evaluate MRA and CTA studies. Second, our study population was relatively small. Further studies with a larger number of patients with SDAVF are needed to clarify the role of these techniques in the clinical setting.

\section{CONCLUSIONS}

In the localization of SDAVF, 3T DCE-MRA was more reliable than 64-CTA. Thus, 3T DCE-MRA may be the first technique for evaluating localization of SDAVF. Additional use of 64-CTA might increase the observer confidence level of the diagnosis.

\section{REFERENCES}

1. Krings T, Geibprasert S. Spinal dural arteriovenous fistulas. AJNR Am J Neuroradiol 2009;30:639-48

2. Van Dijk JM, TerBrugge KG, Willinsky RA, et al. Multidisciplinary management of spinal dural arteriovenous fistulas: clinical presentation and long-term follow-up in 49 patients. Stroke 2002; 33:1578-83

3. Narvid J, Hetts SW, Larsen D, et al. Spinal dural arteriovenous fistulae: clinical features and long-term results. Neurosurgery 2008;62:159-66

4. Rodesch G, Lasjaunias P. Spinal cord arteriovenous shunts: from imaging to management. Eur J Radiol 2003;46:221-32

5. Luetmer PH, Lane JI, Gilbertson JR, et al. Preangiographic evaluation of spinal dural arteriovenous fistulas with elliptic centric contrast-enhanced MR angiography and effect on radiation dose and volume of iodinated contrast material. AJNR Am J Neuroradiol 2005;26:711-18

6. Lai PH, Pan HB, Yang CF, et al. Multi-detector row computed tomography angiography in diagnosing spinal dural arteriovenous fistula: initial experience. Stroke 2005;36:1562-64

7. Zampakis P, Santosh C, Taylor W, et al. The role of non-invasive computed tomography in patients with suspected dural fistulas with spinal drainage. Neurosurgery 2006;58:686-94

8. Yamaguchi S, Nagayama T, Eguchi K, et al. Accuracy and pitfalls of multidetector-row computed tomography in detecting spinal dural arteriovenous fistulas. J Neurosurg Spine 2010;12:243-48

9. Farb RI, Kim JK, Willinsky RA, et al. Spinal dural arteriovenous fistula localization with a technique of first-pass gadolinium-enhanced MR angiography: initial experience. Radiology 2002; 222:843-50

10. Saraf-Lavi E, Bowen BC, Quencer RM, et al. Detection of spinal dural arteriovenous fistulae with MR imaging and contrast-enhanced MR angiography: sensitivity, specificity, and prediction of vertebral level. AJNR Am J Neuroradiol 2002;23:858-67

11. Mull M, Nijenhuis RJ, Backes WH, et al. Value and limitations of contrast-enhanced MR angiography in spinal arteriovenous malformations and dural arteriovenous fistulas. AJNR Am J Neuroradiol 2007;28:1249-58

12. Flohr TG, Schaller S, Stierstorfer K, et al. Multi-detector row CT systems and image-reconstruction techniques. Radiology 2005; 235:756-73

13. Vargas MI, Nguyen D, Viallon M, et al. Dynamic MR angiography (MRA) of spinal vascular diseases at 3T. Eur Radiol 2010;20:2491-95

14. Schmitz BL, Aschoff AJ, Hoffmann MH, et al. Advantages and pitfalls in 3 T MR brain imaging: a pictorial review. AJNR Am J Neuroradiol 2005;26:2229-37

15. DeLano MC, DeMarco JK. 3.0 T versus 1.5 TMR angiography of the head and neck. Neuroimaging Clin North Am 2006;16:321-41

16. Jones DG, Shrimpton PC. Survey of CT Practice in the UK: Normalised Organ Doses for X-Ray Computed Tomography Calculated Using Monte Carlo Techniques. Harwell, United Kingdom: National Radiological Protection Board; 1991

17. Nakaura T, Nakamura S, Maruyama N, et al. Low contrast agent and radiation dose protocol for hepatic dynamic CT of thin adults at 256-detector row CT: effect of low tube voltage and hybrid iterative reconstruction algorithm on image quality. Radiology 2012;264: $445-54$ 